\title{
Il port sottocutaneo in emodialisi: passato, presente, futuro
}

\author{
F. Cavatorta
}

U.O.C. Nefrologia e Dialisi, Imperia, ASL 1 Imperiese

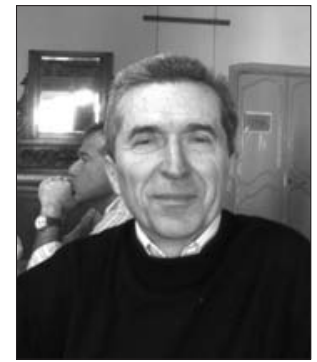

D lice utilizzo nei primi anni Ottanta di sistemi per l'accesso vascolare parzialmente sottocutanei quali l'Hemasite e il Bentley DiaTAP button, negli anni Novanta sono stati messi a disposizione del clinico da parte dell'industria dei dispositivi per emodialisi totalmente impiantati in alternativa ai cateteri venosi centrali (CVC) tunnellizzati.

Questi devices avrebbero dovuto avere vari vantaggi potenziali e pertanto soppiantare in molti casi CVC transcutanei (Tab. I).

I due sistemi sviluppati sono stati il LifeSite Hemodialysis Access System e il Dialock Hemodialysis System.

Attualmente (riferendosi al momento in cui viene redatto questo testo) in Italia non sono disponibili questi dispositivi e l'unico sistema utilizzato soprattutto negli USA - in quanto approvato dalla FDA - è il LifeSite ${ }^{\circledR}$ prodotto dalla ditta Vasca (Vasca, Inc, Tewksbury, MA, USA) (Fig.1).

Si tratta di un sistema costituito da una camera valvolata in titanio collegata a un $\mathrm{CVC}$ da $12 \mathrm{~F}$ in silicone. Il device, che viene impiantato in doppio, utilizza per il suo funzionamento semplici aghi fistola da 14 gauge.

\section{TABELLA I}

Caratteristiche CVC tunnellizzati CVC totalmente
long-term sottocutanei

\begin{tabular}{lll}
\hline Posizione & Transcutanea & Sottocutanea \\
Cuffia sottocutanea & Sì & No \\
Difficoltà nell'impianto & Minore & Maggiore \\
Sede preferenziale & $\begin{array}{l}\text { Vena giugulare } \\
\text { int. destra }\end{array}$ & $\begin{array}{l}\text { Vena giugulare } \\
\text { int.destra }\end{array}$ \\
Tecnica di accesso & Luer-lock & Aghi-dialisi \\
Utilizzo immediato & Sì & Sì \\
$\begin{array}{l}\text { Difficoltà di accesso } \\
\text { al sistema }\end{array}$ & No & Sì (di grado medio) \\
Dolore nel collegamento & No & Sì (di grado medio) \\
Igiene personale & Peggiore & Migliore \\
Gradimento & Basso & Elevato \\
$\begin{array}{l}\text { Incidenza infezioni } \\
\text { del sistema }\end{array}$ & Maggiore & Minore \\
\hline
\end{tabular}




Giornale
di Tecniche
Nefrologiche
\& Dialitiche
Anno XVIII n ${ }^{\circ} 4$
C) Wichtig Editore 2006

\section{TABELLA II - INDICAZIONI AL POSIZIONAMENTO DI PORT}

\section{Trattamento dialitico cronico}

Aspettativa di vita $>1$ anno

Late referral con patrimonio vascolare scarso

Pregresso fallimento di FAV

Esaurimento dell'albero venoso

Pazienti complicati e casi limite

\section{TABELLA III - CONTROINDICAZIONI}

Malformazioni/lesioni delle vene centrali

Coagulopatie

Neoplasie attive

Processi infettivi conclamati

L'altro sistema totalmente impiantato meritevole di ricordo ma non più in produzione, è il Dialock ${ }^{\circledR}$ della Biolink (Middleboro, MA, USA) - costituito da una camera valvolata doppia collegata a due cateteri in silicone rinforzato; l'uso richiedeva aghi de- dicati. Di tale dispositivo ne sono stati impiantati in Italia negli anni scorsi circa una novantina di esemplari con risultati complessivamente soddisfacenti. In un numero imprecisato di pazienti il sistema è ancora funzionante (Fig. 2).

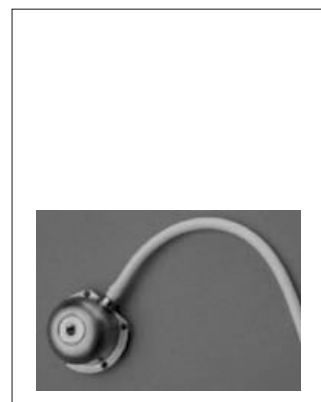

Il port

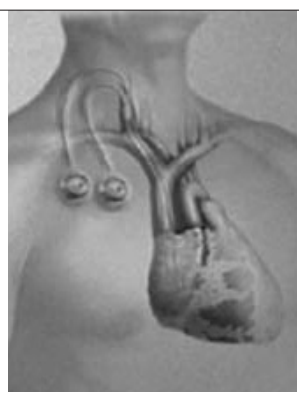

L'impianto

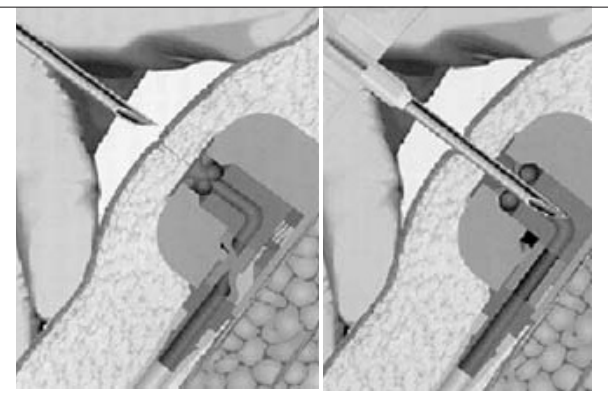

La puntura

Fig. 1 - Il sistema "Vasca - LifeSite ${ }^{\circledR}$ "

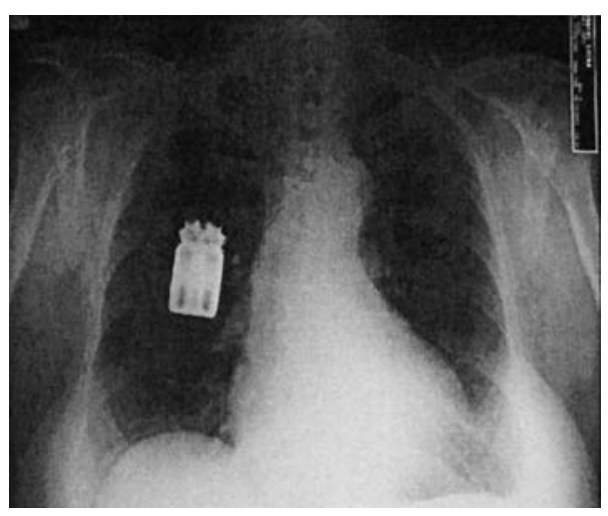

\section{Studio di Sodeman}

\begin{tabular}{lccc}
\hline & $\begin{array}{c}\text { Pazienti } \\
(\mathrm{n})\end{array}$ & $\begin{array}{c}\text { Eventi/ } \\
1000 \text { giorni }\end{array}$ & $\begin{array}{c}\text { Problemi } \\
\text { risolti }\end{array}$ \\
\hline Senza infezione & 44 & & \\
$\begin{array}{l}\text { Emoculture }+ \\
\text { Infez. tasca }\end{array}$ & $93 \%)$ & $\sim$ & $\sim$ \\
& 21 & 0.29 & $100 \%$ \\
\end{tabular}

\section{Studi pilota}

I primi studi condotti con il Dialock sono quelli di Canaud e Levin (Seminar Dial 1999) su 23 pazienti con sopravvivenza a 12 mesi del $71 \%$ e una incidenza di batteriemia pari $2.3 \times 1000 \mathrm{gg}$ e di Sodeman (Blood Purif 2001) su 70 pazienti (vedi Tabella in basso).

\section{Studio Policentrico Italiano}

In base alla esperienza derivante dallo studio Italiano condotto negli anni 2000-2002 e in particolare quella dell'Ospedale G. Bosco di Torino con 50 impianti su 49 pazienti e quella della Nefrologia Imperiese con 32 impianti su 29 pazienti il dispositivo si è dimostrato significativamente più sicuro dei cateteri tunnellizzati tradizionali nei confronti della prevenzione delle infezioni cutanee mentre l'incidenza di batteriemia non è risultata statisticamente

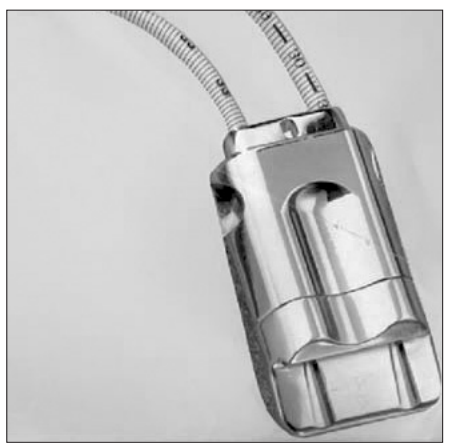

Fig. 2 - Dialock $^{\circledR}$ Biolink

Fig. 3 - Controllo radiografico di Dialock. 
differente.

La puntura del port ha richiesto soprattutto all'inizio un addestramento del personale medico e infermieristico ed in molti casi, sia pure talora solo per alcune settimane, l'uso di una pomata anestetica.

L'accurata gestione del sistema con l'avvertenza di variare il punto di entrata degli aghi nella cute ha consentito di poter mantenere la cute sovrastante in buon stato (Fig. 4).

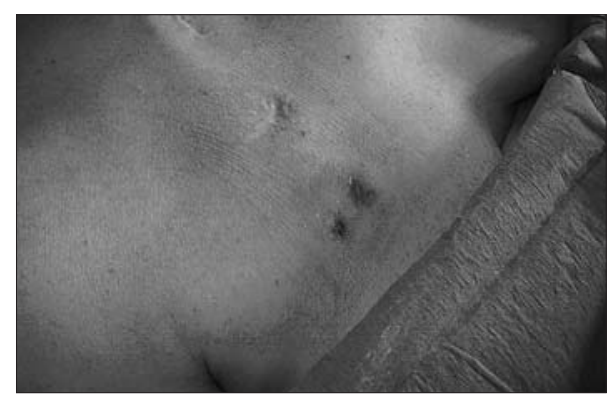

Fig. 4 - Stato della cute dopo 12 mesi di utilizzo del Dialock.

\section{Inconvenienti riscontrati con il sistema Dialock}

\section{- Erosione della cute sopra il port}

In alcuni pazienti la spiccata riduzione dello spessore del sottocute a livello della parete toracica anteriore ha portato alla erosione della cute con conseguente infezione ed inevitabile rimozione del sistema. Per la soluzione di questa problematica era stato messo in commercio un modello di port di minor spessore e peso, pertanto più adatto per pazienti magri (Fig. 5).

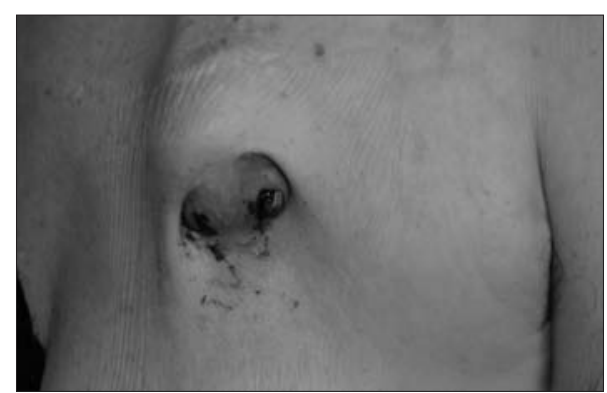

Fig. 5 - Erosione della cute a livello dei siti di venipuntura del port.

\section{- Distacco o rottura del catetere dal port}

Anche se rara questa complicanza si è verificata a seguito di un difettoso ancoraggio dei cateteri al port e soprattutto con i primi modelli di cateteri per una rottura spontanea del silicone in corrispondenza del punto di ingresso nella vena giugulare interna.

\section{- Infezione del port}

Si tratta di una complicanza abbastanza rara, naturalmente evitabile nel rispetto delle necessarie norme di asepsi durante le procedure di attacco e stacco (Fig. 6).

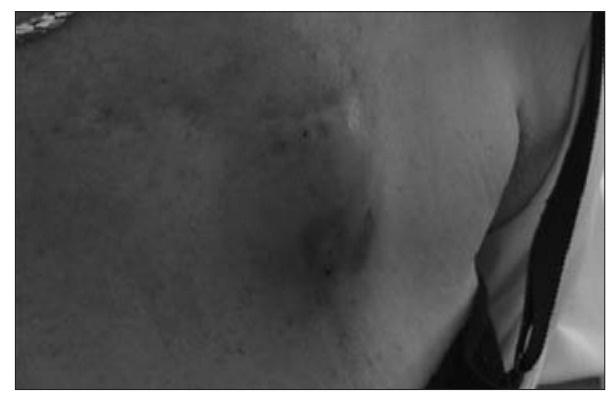

Fig. 6 - Raccolta sottocutanea da ascesso a livello del port.

\section{- Malfunzionamento da dislocazione della punta}

In alcuni pazienti di sesso femminile il malfunzionamento del Dialock dovuto alla dislocazione tardiva dei cateteri ha richiesto interventi correttivi di fissaggio del port alla fascia muscolare del grande pettorale in una sede più alta $\mathrm{e}$ spesso l'utilizzo di un reggiseno adatto a limitare lo spostamento in basso del sistema in posizione eretta.

Bisogna infatti considerare che la sede del tip dei CVC varia con gli atti respiratori e con la posizione del paziente: in posizione eretta il tip risale verso l'alto per la trazione esercitata dai tessuti della parete toracica sull'anello di ancoraggio del catetere e per la discesa del diaframma provocata dal peso dei visceri addominali. La punta del CVC può pertanto subire ampie variazioni di posizione anche di vari centimetri soprattutto nei pazienti obesi e nelle donne con grosse mammelle.

Con il Dialock lo spostamento del CVC in posizione eretta è ancora maggiore dato il maggior peso del port pari a 50 grammi.

In effetti l'unica vera controindicazione a una maggiore diffusione del sistema totalmente impiantabile Dialock è stato l'alto costo di impianto e di gestione.
Il Dialock non ha ottenuto l'approvazione della FDA in USA e in mancanza dell'espansione sul mercato europeo la produzione è stata interrotta; attualmente viene garantita la produzione degli aghi per mantenere la gestione dei port in uso.

Di certo i maggiori costi legati all'inserimento e alla gestione del dispositivo e la maggiore difficoltà di tipo chirurgico nell'impianto hanno limitato la sua diffusione.

\section{Fine dell'avventura oppure i CVC sottocutanei potranno ancora avere un futuro?}

È difficile rispondere a questa domanda; tuttavia se l'industria riuscirà nell'impresa di ridurre i costi del sistema, migliorando nel contempo l'accesso al port e le caratteristiche fisiche dello stesso, quali il peso e le dimensioni, insieme a una maggiore prevenzione delle infezioni, allora i CVC totalmente impiantabili potranno diventare una vera alternativa in determinati casi non solo ai CVC tunnellizzati ma anche all'accesso vascolare tradizionale costituito dalla FAV nativa e/o protesica. 